\title{
Advances in Stem Cell Technologies for Disease Therapy: A
}

\section{Current Review}

\section{Nadiya $\mathrm{P}^{*}$}

De Montfort University, Gateway House, Leicester, England

*Corresponding author: Nadiya Patel, De Montfort University, Gateway House, Leicester, England, LE1 9BH.

\section{Review Article}

Volume 1 Issue 1

Received Date: October 03, 2018

Published Date: November 08, 2018

DOI: $10.23880 /$ aabsc- 16000107

\section{Abstract}

Stem cells have the capability of differentiating into limitless cell types, alongside the function of exceptional proliferative capacity. There are three main types of stem cells: embryonic stem cells (ESCs), induced pluripotent stem cells (IPSCs) and mesenchymal stem cells (MSCs). ESCs are highly versatile and hold great therapeutic potential but have great ethical barriers and considerations that are yet to be overcome. IPSCs have become increasingly popular within research as they are not restrained by any ethical issues and do not require approval for their usage. The aim of this review was to expand on the background and therapeutic potential of ESCs and IPSCs whilst linking this to their use within disease therapy with a specific focus on ethics, tumorigenesis and survivability. The analysis found some conflicting results and a delay in the advance of overcoming the problems of tumorigenesis and survivability of stem cells. Both stem cells types have shown good efficacy but do also come with their disadvantages.

Keywords: Stem Cell; Technologies; Pluripotent stem cells; Embryoblast

\section{Introduction}

Stem cells are undifferentiated cells of a multicellular organism. They can function to self-renew and are capable of indefinitely replicating more cells of the same kind, from which other various types of cells may arise via differentiation. There are three main types of stem cells: embryonic stem cells (ESCs), induced pluripotent stem cells (IPSCs) and mesenchymal stem cells (MSCs). The origin and function of these stem cells varies but they all hold diagnostic and therapeutic potential.

At present, there is extensively rigorous scientific research being carried out into the prospective use of stem cell based treatments for a range of worldwide diseases that currently have poor treatment options.
There are over 600 neurological disorders affecting the nervous system. This includes degenerative diseases, where nerve cells become damaged or eradicated, such as Parkinson's disease and Alzheimer's disease. Collectively, both of these diseases affect 15.7 million people worldwide. A potential route of treatment is through the use of stem cells that have the capability of differentiating into healthy tissue, replacing any lost through disease manifestation and thus justifying the avoidance of lifelong expensive treatments. The use of stem cells may propose the possibility of a renewable source of replacement cells and tissues, leading to the potential treatment of various other diseases including spinal cord injury, heart disease, diabetes, arthritis and macular degeneration. This leads to an understanding that the use of stem cells for tissue repair in a vast range of currently incurable and poorly 
treated conditions may enable advances in these areas and allow for breakthroughs within scientific research.

However, much in depth research including clinical trials has been continuously stuck at a "promising" stage for the last 15 years. This is further justified with there being diminutive change in the number of FDA approved stem cell therapies in the last 8 years. One of many reasons for this is due to the adverse effects of stem cell therapy being unknown and not fully listed due to the short timeframe of clinical trials [1]. Results from clinical trials proving the safety and effectiveness of stem cell based therapies may take a few more years, by which time more individuals may become diagnosed with conditions that could benefit from such research. Currently, bone marrow transplantation is the only stem cell based therapy that is widely used and is proven to be safe and effective. Blood-forming stem cells found in the bone marrow were the first to be discovered and to be used in clinics to aid thousands of patient's worldwide suffering from leukaemia.

This review holds an aim to expand on the background and therapeutic potential of embryonic stem cells (ESCs) and induced pluripotent stem cells (IPSCs) whilst linking this to their use within disease therapy with a specific focus on ethics, tumorigenesis and survivability. An indepth analysis and critical evaluation of recent articles and research in this area will be carried out in order to gain a deeper understanding and provide a conclusion.

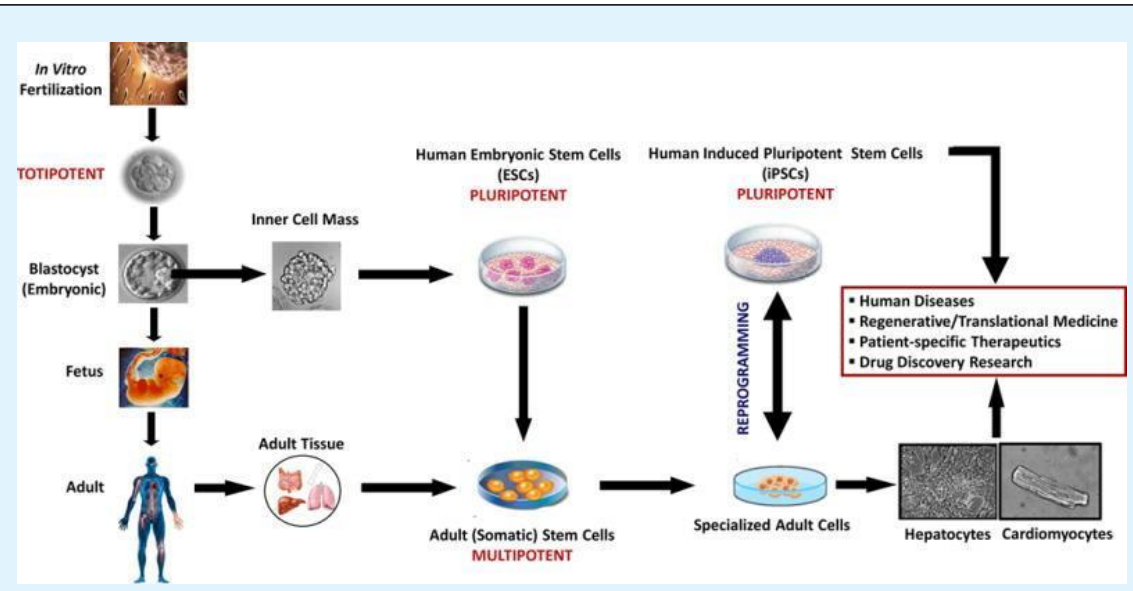

Figure 1: A summary of the potential uses and applications of stem cell technology in diagnostic research. Photo credit: Hansel, et al. [2].

\section{Methodology}

A bibliographical search was performed using NCBI PubMed for journal articles and studies that showed evidence of suitability within the criteria of stem cell technologies for disease therapy. The following keywords were used: "stem cell therapy", "embryonic stem cell therapy", "induced pluripotent stem cell therapy" and "stem cell research". Articles and studies that held a specific focus on stem cell uses and therapeutic potential were chosen as these held the most relevance. All relevant articles from January 2005 to March 2018 were included but any articles published before January 2005 were excluded and disregarded. This was so that the results reflected only recent advances that have been made in stem cell therapy. The methodology was done in a specific manner so that if another person was to replicate the steps, they may obtain the same or very similar findings.

\section{Embryonic Stem Cells}

ESCs are pluripotent stem cells that are derived from the inner cell mass of a blastocyst embryo [3]. Human embryos reach this stage approximately 4-5 days after fertilisation, by which they are composed of 50-150 cells. They contribute remarkable differentiation and proliferative capacities, making them a crucial therapeutic target. Advances made with ESCs in therapy initially started with small successful trials, leading to wider research being established.

In order to obtain ESCs for research, the embryoblast requires isolation which results in the destruction of the blastocyst itself. This raises major ethical concerns, with a particular focus on whether embryos at the preimplantation stage hold the same legal and moral status as embryos in the post-implantation stage. In addition to 
this, ethical issues regarding embryo destruction has led to the implementation of laws restricting their use for research. Debates in the United States questioning when exactly human life begins, have led to a controversial understanding of embryo destruction, linking closely to debates regarding abortion [4]. Problems surrounding ethics have been identified as the main limiting barrier for the use of ESCs in therapy [5]. Notwithstanding this, scientific research with the use of embryos and ESCs has continued in countries that have more relaxed laws regarding the use of embryos alongside private funding [6].

Theoretically, ESCs have a vast potential in terms of regenerative ability, but this is not always reflected in the clinical setting.

Research by Sakai and Andersson [7] tested the use of ESCs in vivo for the treatment of intervertebral disc therapy. Results evidenced that ESCs lacked survivability and adaptivity in the avascular niche of intervertebral disc therapy, displaying little value in this research area. This research was further supported by Mihic, et al. [8] who identified the same obstacles of survivability and tumorigenicity when ESCs were transplanted into heart tissue of animal models. Both researchers highlighted the same efficacy problems with direct use of ESCs as stem cell therapy, although they were applied to different tissues and the origin varied from human to animal. Unsupportive results in animal models, have not aided in favour of the use of ESCs in humans.

A small-scale study by Shroff, et al. [9] into the therapeutic potential of ESCs in cerebral palsy patients has proven to be somewhat useful. Research was undertaken to evaluate the efficacy and safety of ESCs therapy in 91 cerebral palsy patients. Results showed a general improvement in gross motor function and that the use of ESC is effective and safe. As the patient number was small in comparison to the estimated 8000 babies born with cerebral palsy each year, results cannot be generalised without further justification. Also, results were based on an individual rated Gross Motor Function Scale, which may lead to issues with subjectivity and do not provide any details on the biological process underlying the therapeutic uses of ESC in cerebral palsy.

\section{Induced Pluripotent Stem Cells}

IPSCs are pluripotent stem cells that can be directly generated from normal adult cells. They require exposure to specific transcription factors in order to manipulate and revert them back into a pluripotent state where they can be further cultured and used in treatment [10]. IPSCs do not face ethical limitations as they are not obtained from embryos and can be limitlessly quantified from human cells into almost any tissue which can then be used for autologous repair. However much like ESCs, IPSCs have a risk of instability and tumorigenesis which has been highlighted in recent research.

Comparative research by Ronaghi, et al. [11] on the use of ESCs and IPSCs for spinal cord therapy, indicate that IPSCs hold a futuristic role for autologous cell transplantation, whilst being aware of the potential of tumorgenicity. Research on an animal model for spinal cord therapy by Nori, et al. [12] analysed safety concerns of the use of IPSCs, resulting in an understanding that although safety regulations are of paramount importance, the ease of using IPSCs rather than ESCs for treatment is incredibly beneficial. It must be emphasised that both Nori, et al. [12] and Wilson, et al. [13] both conclude that IPSC treatment of almost any damaged tissue is more beneficial when considering the ethical and research issues of ESCs. However, a main barrier hindering greater adoption of IPSCs in the clinical setting is the formation of tumours by IPSCs when they have been transplanted in vivo. According to Gutierrez-Aranda, et al. [14], the genomes of IPSCs may be uncontrollably and inexplicably more unstable as the transcription factors used to transform them may lead to genomic interference. This may potentially limit the usefulness of IPSCs as the specific level and type of genomic interference would require further, secondary research.

Specific research by Hansel, et al. [15] indicates the potential uses of IPSCs in liver diseases including chronic hepatitis and acute liver failure. Hepatocyte transplantation was proposed as an alternative to liver transplantation in patients in order to provide more stability and prolong their lives. However, routine use proposes a challenge as there is a shortage of liver donors to extract hepatocytes from. To combat this, the role of IPSCs was considered and proved useful in the context of hepatic differentiation.

There is currently no medical screening of IPSC cells prior to transplantation, but various novel methods have been identified and may be adopted in the future. The use of FACS based flow cytometry as designed by Hentze, et al. [15] involve the elimination of rogue unidentifiable cells from IPSC cultured population and has produced positive results. These unidentifiable cells are thought to express cell-surface markers and may correlate with tumorigenicity. The removal of them from IPSCs before transplantation showed a significant reduction in teratoma production. 


\section{Conclusion}

The use of ESCs is seemingly extensive but is hindered due to strict ethical laws and regulations that are unlikely to be waivered or overlooked. Thus, the future of stem cell based therapeutic techniques is more likely to be based upon IPSCs due to the lack of ethical considerations and the ease of obtaining the cells. IPSCs require further research in clinical trials to justify their use and more medical screening methods must be put in place in order to reduce the risk of any side effects of their usage. Novel research which may be applied to animal models would be required in order to identify techniques that hold the greatest efficacy which can then be translated therapeutically to humans. The results of stem cell based research now will aid the future of stem cell based therapy for patients of multiple various diseases.

\section{References}

1. Marks P, Witten C, Califf R (2017) Clarifying Stem-Cell Therapy's Benefits and Risks. New England Journal of Medicine 376(11): 1007-1009.

2. Hansel M, Davila J, Vosough M, Gramignoli R, Skvorak K, et al. (2016) The Use of Induced Pluripotent Stem Cells for the Study and Treatment of Liver Diseases. Current Protocols in Toxicology 67: 14.13.1-14.13.27.

3. Baldwin $\mathrm{T}$ (2009) Morality and human embryo research. Introduction to the Talking Point on morality and human embryo research. EMBO reports 10(4): 299-300.

4. Lo B, Parham L (2009) Ethical Issues in Stem Cell Research. Endocrine Reviews 30(3): 204-213.

5. Sugarman J (2008) Human Stem Cell Ethics: Beyond the Embryo. Cell Stem Cell, 2(6), pp: 529-533.

6. Trounson A, McDonald C (2015) Stem Cell Therapies in Clinical Trials: Progress and Challenges. Cell Stem Cell 17(1): 11-22.

7. Sakai D, Andersson G (2015) Stem cell therapy for intervertebral disc regeneration: obstacles and solutions. Nature Reviews Rheumatology 11(4): 243 256.
8. Mihic A, Li J, Miyagi Y, Gagliardi M, Li S, Zu J, et al. (2014) The effect of cyclic stretch on maturation and 3D tissue formation of human embryonic stem cellderived cardiomyocytes. Biomaterials 35(9): 27982808.

9. Shroff G, Gupta A, Barthakur J (2014) Therapeutic potential of human embryonic stem cell transplantation in patients with cerebral palsy. Journal of Translational Medicine 12: 318.

10. Takahashi K, Yamanaka S (2006) Induction of Pluripotent Stem Cells from Mouse Embryonic and Adult Fibroblast Cultures by Defined Factors. Cell 126(4): 663-676.

11. Ronaghi M, Ercegm S, Moreno-Manzano V, Stojkovic M (2010) Challenges of Stem Cell Therapy for Spinal Cord Injury: Human Embryonic Stem Cells, Endogenous Neural Stem Cells or Induced Pluripotent Stem Cells?. Stem Cells 28(1): 93-99.

12. Nori S, Okada Y, Nishimura S, Sasaki T, Itakura G, et al. (2015) Long-Term Safety Issues of iPSC-Based Cell Therapy in a Spinal Cord Injury Model: Oncogenic Transformation with Epithelial-Mesenchymal Transition. Stem Cell Reports 4(3): 360-373.

13. KD W, JC W (2015) Induced pluripotent stem cells are induced pluripotent stem cell-like cells. Journal of biomedical research 29(1): 1-2.

14. Gutierrez-Aranda I, Ramos-Mejia V, Bueno C, MunozLopez M, Real P, et al. (2010) Human Induced Pluripotent Stem Cells Develop Teratoma More Efficiently and Faster Than Human Embryonic Stem Cells Regardless the Site of Injection. STEM CELLS 28(9): 1568-1570.

15. Hentze H, Soong P, Wang S, Phillips B, Putti T, et al. (2009) Teratoma formation by human embryonic stem cells: Evaluation of essential parameters for future safety studies. Stem Cell Research 2(3): 198210.

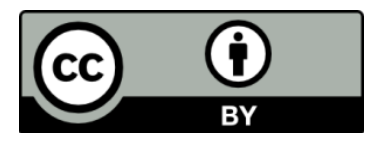

